HCBS type is associated with nursing home readmission, but not global life satisfaction 12 months after transitioning into the community. Participants with PCA and 24-hour live-in services were less likely to be readmitted to nursing home at 12 months compared to those with traditional HCBS. Results from this study have implications for improving HCBS delivery for older adults living in the community.

\section{PATHS TO POSITIVITY: AGE DIFFERENCES IN APPRAISALS OF CONTROL AND THEIR CONTRIBUTION TO EMOTIONAL EXPERIENCE}

\section{N. Young, DePaul University}

Research suggests that older adulthood is a time of increasing losses in several domains, which decreases control capacity relative to younger adulthood. Despite these losses, older adults also experience increased emotional well-being compared to younger adulthood. This "paradox of aging" is counter to research that suggests losses of control lead to worse emotional well-being, and point toward the importance of evaluations of control, rather than capacity. The current study used ambiguous scenarios to investigate the relationship between evaluations of control and emotion between younger and older adulthood. Older and younger participants read fourteen ambiguous scenarios and after each scenario they appraised them on three different control dimensions: self-control, other-control, and circumstantial-control. Afterward, they rated their feelings. The results showed that older adults had greater differences between self-, other-, and circumstantial-control relative to younger adults $(\mathrm{F}(2,196)=16.37, \mathrm{p}<.001 \mathrm{n} 2=.086)$, and that older adults experienced less negative emotion $(t(98)=4.03, p<.001, d=.81)$, but equal positive emotion compared to younger adults $(\mathrm{t}(98)=-.662, \mathrm{p}=.51, \mathrm{~d}=-.13)$. More importantly, we found that appraisals of control eliminated age-related differences in emotional experience $(\mathrm{F} / 1$, $95)=3.29, \mathrm{p}=.073, \mathrm{n} 2=.026)$, suggesting that age differences in emotional experience are related to age differences in control appraisals. These findings reflect the importance of considering the role of appraisal processes in age differences in emotional experience.

\section{PERCEPTIONS OF RISK FACTORS FOR ALZHEIMER'S DISEASE AMONG COMMUNITY LIVING, NON- DEMENTED OLDER AFRICAN AMERICANS. \\ C. Glover ${ }^{1}$, B. James ${ }^{2}$, S. CoCroft ${ }^{3}$, L. Barnes ${ }^{1}, 1$. Rush Alzheimer's Disease Center, 2. Rush University, 3. Duke University}

Heightened Alzheimer's disease (AD) risk among African Americans represents a racial disparity in aging. An open question remains about variables that older African Americans perceive as AD risk factors. This study examines perceptions of potential AD risk factors among older nondemented African Americans. Participants from the Minority Aging Research Study ( $N=610$; mean age $=74.6$ years, mean education $=14.9$ years, and $24 \%$ men) indicated how important 9 factors were in increasing one's risk of AD using a 4-point Likert scale from $1=$ not at all important to $4=$ extremely important. We examined perceptions of AD risk factors as a function of age, education, gender, and global cognition using separate logistic regression models for each factor. Of the 9 AD risk factors, genetics and God's Will were significantly related to predictors of interest. Younger participants (est. $=-0.06, \mathrm{p}=0.02$ ) and those with more education (est. $=0.14, \mathrm{p}=0.02$ ) were more likely to report genetics as extremely important. Participants with more education were less likely to indicate God's Will as extremely important (est. $=-0.14, \mathrm{p}<.0005)$. Genetics and God's Will are perceived as salient risk factors for $\mathrm{AD}$ among subgroups of older African Americans. Younger and more educated African Americans perceive genetics as an important AD risk factor while those with less education perceive God's Will as an important AD risk factor. As older African Americans are not a monolithic group, findings can facilitate the design of effective and culturally competent educational tools for meaningful engagement with older African Americans about AD.

\section{PERSONALITY CHANGES WITH DEMENTIA FROM THE INFORMANT PERSPECTIVE: NEW DATA AND META-ANALYSIS}

A. Terracciano ${ }^{1}$, M. Islam ${ }^{1}$, M. Mazumder ${ }^{1}$, D. SchwabeWarf $^{1}$, Y. Stephan ${ }^{2}$, A. Sutin 1 , 1. Florida State University,

\section{University of Montpellier}

We examined changes in personality in individuals with mild cognitive impairment (MCI) or dementia as observed by family members using both new data and a meta-analysis of the published literature. Similar to past studies, we obtained current and retrospective personality assessments of individuals with MCI or dementia by family informants $(\mathrm{n}=50)$. In the new sample, the five major dimensions (neuroticism, extraversion, openness, agreeableness, and conscientiousness) and facets of personality were assessed using the first-half NEO Personality Inventory-3. In the new sample and meta-analysis (18 samples; $\mathrm{n}=542$ ) we found consistent changes in personality from the premorbid to current state in patients with cognitive impairment. The largest changes ( $>1$ SD) were declines in conscientiousness (particularly for the facets of self-discipline and competence) and extraversion (decreased energy and assertiveness), as well as increases in neuroticism (increased vulnerability to stress). The new sample suggested that personality changes were larger in individuals taking cognition-enhancing medication (cholinesterase inhibitors and/or memantine). More recent studies and those that examined individuals with MCI found smaller effects. Despite the large systematic meanlevel changes, test-retest coefficients remained moderate to high, which suggest that individual differences in personality are relatively maintained, at least at the stage of dementia examined in this study. In conclusion, and consistent with the clinical criteria for the diagnosis of dementia, the new study and meta-analysis found replicable evidence for large changes in personality among individuals with MCI or dementia.

\section{PET OWNERSHIP AND SELF-REPORTED HEALTH: DOES THE TYPE OF PET MATTER?}

\section{N. Pitheckoff, University of Massachusetts Boston}

Pets are an important part of many individuals' lives. Approximately, $68 \%$ of Americans own some type of pet (American Pet Products Association, 2018). Research has generally shown that pets may have positive physiological, psychological, and social impacts on human health. Using 